\title{
A Coalitional Game Mechanism of Tasks Execution Considering Trust Relationship among CRPs in Clouds
}

\author{
Lijun $\mathrm{Xu}$ and Yanjun Liu \\ School of Computer and Information Engineering, Xinxiang University, Xinxiang \\ 453003, P.R. China \\ 1450384751@qq.com,xulj_123@sina.com
}

\begin{abstract}
Cloud Resource Providers (CRPs) can form CRPs coalitions for executing large scale application tasks by pooling their resources together in cloud computing. However, forming such CRPS coalitions is a challenging problem especially when the trust relationships among CRPs have to be taken into account. Aiming at this problem, we model the formation of CRPs coalitions by considering the trust and reputation of the participanting CRPs in clouds in this paper. An algorithm TCFA for coalition formation is proposed, which can enable the CRPs with high reputation to join into a coalition reducing the cost of execution and guaranteeing the maximum profit for the participanting CRPs. Furthermore, we prove that the formed coalition determined by TCFA is stable, which means that the CRPs that are the part of the coalition do not have incentives to break away from it. Meanwhile, we prove that TCFA can produce a Pareto optimal solution. The results of simulation experiments show that our algorithm TCFA not only can produce stable coalitions structure composed of CRPs with higher reputation, but also can obtain higher individual profit among coalitional members.
\end{abstract}

Keywords: Cloud computing, Tasks scheduling, Coalitional game, Trust relationship

\section{Introduction}

Cloud computing system are composed of heterogeneous resources owned by autonmous data centers [1]. The resource management in such open environment is a very challenging problem [2]. Undoubtedly, efficient resource management could lead to efficient resource utilization and better execution of application tasks in clouds. One important method of resource management in clouds is that Cloud Resource Providers (CRPs) can pool their resources together for faster executing large-scale application tasks, i.e., they can collaborate with each other and form CRPs coalitions. However, CRPs' reliability of executing the cloud tasks is a problem in the formation of CRPs coalitions. In some cases, a CRP agrees to provide some resources, but it fails to deliver the promised resources to a CRPs coalitions finally. As a result, the cloud tasks could not be executed by the coalition on schedule. So, the trust among CRPs based on their behaviors have to be considered, which can avoid the above problem in CRPs coalition formation. CRPs always desire to establish CRPs coalitions with the most trusted CRPs and obtain higher profit. A CRP allocates a trust value to another CRP based on their past interactions. Trust is based on direct interactions of CRPs. If some CRPs have no past interactions with another CRP, they can use the reputation of that CRP to evaluate how likely it is to provide the requested resource. This reputation is the opinion of other CRPs that have had direct interactions with both CPRs to evaluate how likely they are to prvide the requested resource. Then, a CRP's global reputation can be defined as how likely is this CRP to provide the requested resource based on the opinion of all CRPs. In this 
paper, an algorithm for the formation of CRPs coalitions that takes into account the reputation of each of the CRPs that form the coalition is presented.

The trust is a key in utility computing environment such as grid computing and cloud computing, especially when executing application tasks from public users. Trusted computing model can improve the cloud computing security and will not bring much complexity to users [3,4]. In [5], a novel cloud service-composition method based on the trust span tree was proposed. The trust relationship can be built after many times credible interaction between services requester and provider, and the trust union would be formed through the credible relationship evolution. In order to protect the security of cloud entities and better practice cloud's objectives of providing low-cost and on-demand services, [6] proposes a novel cloud trust transaction framework and a new trust fuzzy comprehensive evaluation based cloud service discovery algorithm. The new algorithm uses trust multi-dimensional vector to represent the credibility of providers when offer different types of services and applies fuzzy comprehensive evaluation method to classify services. [7] proposes an energy-aware and trust-driven virtual machine scheduling TD energy-aware-opt algorithm adapting to cloud. This algorithm matchs the tasks and virtual machine by the trust mechanism between resources and tasks' reached the purpose of ensuring the user task performance and energy-aware in data centers. In [8], based on the grid trust model and trust utilization function, a trust-driven grid job scheduling problem was studied and trust-driven Min_Min and Sufferage job scheduling heuristic was proposed. [9] proposes a method in which a GSP decides to allocate resource based on the combination of the trust value and the bidding price of a requester, called Qgrid. Qgrid is based on Q-learning techniques that balance the relative importance of trust and price. Although the trust is considered, our work in this paper differs from these works [5-9], in which the CRPs can lead to more efficiently utilization of resources and better execution of application tasks in clouds.

The cooperation among CRPs is an efficient way for obtaining more profits when executing the application tasks from cloud users. [10] presents a game model of cloud resource allocation based on QoS requirements, in which QoS of resource allocation is formulized as a cooperative game problem and the Pareto optimality solution satisfying QoS demand is proved to exist. To maximize the benefit of the mobile cloud service providers, [11] proposes a framework for resource allocation to the mobile applications, and revenue management and cooperation formation among service providers. For resource allocation to the mobile applications, she formulates and solves optimization models to obtain the optimal number of application instances that can be supported to maximize the revenue of the service providers while meeting the resource requirements of the mobile applications. Based on the revenue shares, the mobile cloud service providers can decide whether to cooperate and share the resources in the resource pool or not. Also, the provider can optimize the decision on the amount of resources to contribute to the resource pool. Similar as [11], [12] also considers a mobile cloud computing environment in which the service providers can form a coalition to create a resource pool to support the mobile application. For a given coalition of service providers, the revenue obtained from utilizing the resource pool has to be shared among the service providers. A coalitional game model is developed for sharing the revenue. [13] studies the cooperative behavior of multiple cloud providers and the hierarchical cooperative game model is presented. Given a coalition of cloud providers, the resource and revenue sharing of a resource pool is presented. To obtain the solution, the paper develops the stochastic linear programming game model which takes the uncertainty of internal users from each provider into account. However, these works ignore the trust relationship among CRPs when they form coalitions.

Different from the above works [5-13], we study the problem of CRPs coalition formation in cloud computing considering the trust relationship among CRPs when they execute large scale application tasks in this paper. We present an algorithm TCFA that 
can allow the CRPs to make their own decisions to join into coalitions and determine the mapping of the tasks to each of the coalitions that minimizes the cost of execution by using our proposed algorithm MIN_COST. In addition, our algorithm can guarantee the highest reputation among its participating CRPs and maximizes the individual payoff of its members.

\section{Tasks Execution Model in Clouds}

A cloud user desires to execute a application tasks $\mathbf{T}=\left\{T_{1}, T_{2}, \ldots, T_{n}\right\}$, comprising $n$ independent tasks, on the available set of Cloud Resource Providers (CRPs) by a given deadline $D$. Each task $T \in \mathbf{T}$ composing the application program is characterized by its workload $M I(T)$, unit: Million Instructor. We assume that executing task $\mathbf{T}$ requires a large number of resources which can not be provided by a single CRP. Thus, several CRPs should form a coalition to execute tasks. We consider that a set of $\mathrm{m}$ CRPs, $\mathbf{R}=\left\{R_{1}, R_{2}, \ldots, R_{m}\right\}$, are available and are willing to provide resources for executing tasks. All CRPs are assumed to be self-interested and welfare-maximizing entities. Each CRP owns several resouces which are abstracted as a single host with the capacity $\operatorname{MIPS}(R)$, unit: Million Instructor Percent Second. Then, the execution time of task $T$ at CRP $R$ is given by the execution time function $t: \mathbf{T} \times \mathbf{R} \rightarrow \mathbf{R}^{+}$, where $t(T, R)=\operatorname{MI}(T) / M I P S(R)$. When executing a task, a CRP incurs some cost, which is given by the cost function $c: \mathbf{T} \times \mathbf{R} \rightarrow \mathbf{R}^{+}$. A user will pay a price $P$ less than her available budget $B$ if the task is executed to completion by deadline D. In order to have necessary resources to execute tasks, CRPs should form CRPs coalitions to maximize their profit, which is defined as the difference between the payment received by a CRP and its execution cost. In other words, if the profit is negative, the CRP will choose not to participate.

\section{Trust Model Based on Weighted Directed Graph}

The weighted directed graph theory will be used to describe the trust model in our CRPs coalitions formation. A CRP assigns a trust value to another CRP based on their interactions. The trust relationship among CRPs is defined as a weighted directed graph $(\mathbf{R}, \mathbf{E})$, where $\mathbf{R}$ is the set of CRPs that represents the vertices in the graph and $\mathbf{E}$ is the set of edges. The weight $w_{i j}$ associated with edge $(i, j)$ represents the amount of trust that $R_{i}$ assigns to $R_{j}, R_{i}, R_{j} \in \mathbf{R}$. The weight $w_{i j}$ is the strength of the trust relationship from $R_{i}$ to $R_{j}$ which is based on past interactions among them, which means that if $w_{i j}=0$, then $R_{i}$ distrusts $R_{j}$ completely. Figure 1 shows the trust model among CRPs repressed by a weighted directed graph.

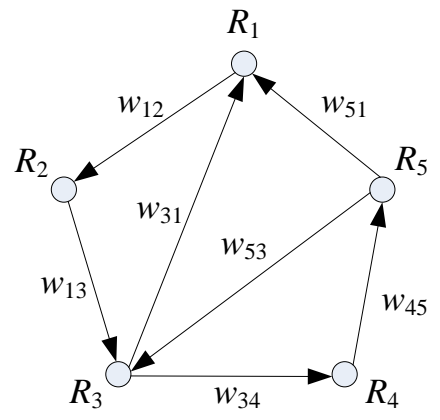

\section{Figure 1. Trust Model among CRPs}

For executing tasks efficiently, $R_{i}$ should select $R_{j}$ based not only on their direct trust $w_{i j}$ but on the trust the other CRPs have on $R_{j}$ when form a CRPs coalition. In other words, apart from the direct trust, the reputation of CRPs have to be taken into account, which 
can measure how likely is the CRP to provide the requested resources based on all other CRPs' opinions. The direct trust among CRPs can be used for local ratings. To assign a single rating to a CRP, i.e., the local trust value, the normalization of the direct trust is used in such a way that the values of local trust are between 0 and $1 . \sigma_{i j} \in[0,1]$ denotes the normalization trust. $\sum_{j=1}^{m} \sigma_{i j}=1$ for $i=1,2, \ldots, m, m$ is the number of CRPs. Then,

$\sigma_{i j}=\frac{w_{i j}}{\sum_{k \in N_{i}} w_{i k}}, N_{i}=\left\{R_{j} \mid \exists(i, j) \in \mathbf{E}\right\}$

Next, we will discuss how to determine the global reputation of each CRP within a given set $\mathbf{R}$ of CRPs. $r_{R_{i \rightarrow j}}^{l}$ denotes the trust that $R_{i}$ assigns to $R_{j}$ based on the opinion of $l$ CRPs. Let A denote the matrix of normalized trust of the graph $(\mathbf{R}, \mathbf{E})$, where $\sigma_{i j} \in[0,1]$ denotes the normalization trust. To find the reputation between CRPs $R_{i}$ and $R_{j}, R_{i}$ uses its neighbors opinions about $R_{j}$ by weighted their opinion using the trust $R_{i}$ places on them:

$r_{R_{i \rightarrow j}}^{1}=\sum_{R_{k} \in \mathbf{R}}\left(\sigma_{k j}\right)^{T} x_{R_{i \rightarrow k}}^{0}$

The above approach can aggregate the local trust values of all CRPs and compute the reputation of CRPs using the transitive property of the trust, which can facilitate trust propagation and trust aggregation. This procedure can be done for the neighbors of neighbors, which can improve the accuracy of trust propagation.

$r_{R_{i \rightarrow j}}^{l}=\sum_{R_{k} \in \mathbf{R}}\left(\sigma_{i j}\right)^{T} r_{R_{i \rightarrow k}}^{l-1}$

$\mathbf{r}_{R_{i \rightarrow j}}^{l}$ denotes the vector that contains all the reputation values that $R_{i}$ assigns to the other CRPs using $l$ CRPs. In other words, the length of a path from $R_{i}$ to other CRPs in the graph is $l$. Using the matrix notation, equation 4 for all CRPs $R i, i=1,2, \ldots, m$ can be rewritten as follows:

$\mathbf{r}_{R_{i}}^{l}=A^{T} \mathbf{r}_{R_{i}}^{l-1}$

If $l$ is large, $R_{i}$ will assign a reputation value to each CRP considering the opinion of all CRPs. If all other CRPs do the same to find the reputation values of all CRPs, they will find the same reputation values as in $\mathbf{r}_{R_{i \rightarrow j}}^{l}$. As a result, $\mathbf{r}_{R_{i \rightarrow j}}^{l}$ converges to the global reputation of the CRPs. This vector is the left principal eigenvector of $A$, that is, it satisfies:

$\lambda \mathbf{r}=A^{T} \mathbf{r}$

Where $\lambda$ is the eigenvalue of $A$. then, this procedure determine the global reputation of each CRP. By using this method, we can convert the trust values between each pair of CRPs into a global reputation for each CRP. $r_{i}$ in $\mathbf{r}$ gives the global reputation value of $R_{i}$. $\mathbf{r}$ determines the centrality of the CRPs based on their reputation.

The average global reputation for a set of CRPs $\mathbf{R}$ is defined as

$\bar{r}(\mathbf{R})=\frac{1}{|\mathbf{R}|} \sum_{i: R_{i} \in \mathbf{R}} r_{i}$

It will be used as a metric to characterize the aggregate reputation of the members of the CRPs coalition.

\section{Formation of CRPs coalition in Clouds}

The coalitional game theory is used to model the CRPs coalition formation problem. A coalitional game is defined by the pair $(\mathbf{R}, v)$, where $\mathbf{R}$ is the set of players (CRPs) and $v$ is the characteristic function, defined on $v: S \subseteq \mathbf{R} \rightarrow \mathbf{R}^{+}$such that $v(\varnothing)=0$. 
Each subset $S \subseteq \mathbf{R}$ is a coalition. A CRPs coalition has a value given by $v(S)$ representing the profit obtained when the members of a coalition work as a group. To maximize the value of a coalition, a CRP prefers to join a coalition with higher value and members of a coalition prefer to join with CRPs that have higher reputation values. The CRPs coalition formation not only depends on profit, but it also depends on how much trust the CRPs that are part of the coalition have on each other. The reputation of CRPs in a coalition means how much reputation each CRP has based on the opinion of all CRPs in that coalition. The trust graph among the CRPs in a coalition has an impact on the formation of the coalition. We define a subgraph $(S, \Xi)$ of $(\mathbf{R}, \mathbf{E}), \mathrm{S}$ is the set of CRPs in coalition and $\Xi$ is a set of edges among CRPs in S. As is the matrix containing the trust values of the CRPs in S. we model the coalition formation based on reputation as a coalition formation problem. Coalition formation is the partitioning of the players into disjoint sets. A coalitional structure $W=\left\{S_{1}, S_{2}, \ldots, S_{h}\right\}$ forms a partition such that each player is a member of exactly one coalition, i.e., $S_{i} \cap S_{j}=\emptyset$ for all $\mathrm{i}$ and $\mathrm{j}$ where $i \neq j$ and $\cup_{S i \in W} S_{i}=\mathbf{R}$.

For each coalition composed of CRPs from $\mathbf{R}$, there exists a mapping $\pi_{s}: \mathbf{T} \rightarrow S$, which assigns task $T \in \mathbf{T}$ to CRP $R \in S$. To make sure that a coalition is able to execute $\mathbf{T}$, we need to find a mapping of all tasks on the members of the coalition in such a way that the mapping satisfies all constraints. This problem is known as the tasks scheduling problem.

The tasks scheduling problem finds a mapping of the $n$ tasks of cloud user to $k$ CRPs in the coalition $S$ where $k=|S|$. we consider the following decision variables:

$\chi_{S}(T, R)= \begin{cases}1, & \pi_{S}(T)=R \\ 0, & \pi_{S}(T) \neq R\end{cases}$

The tasks scheduling problem can be defined as the follows optimization problem,

Objective function

$\operatorname{Min} C(\mathbf{T}, S)=\sum_{T \in \mathbf{T}} \sum_{R \in S} \chi_{S}(T, R) c(T, R)$

Subject to

$\left\{\begin{array}{l}\sum_{T \in \mathbf{T}} \sum_{R \in S} \chi_{S}(T, R) c(T, R) \leq P \\ \sum_{T \in \mathbf{T}} \chi_{S}(T, R) t(T, R) \leq D \\ \sum_{R \in S} \chi_{S}(T, R)=1 \\ \sum_{T \in \mathbf{T}} \chi_{S}(T, R) \geq 1 \\ \chi_{S}(T, R) \in\{0,1\} \\ \forall R \in S, T \in \mathbf{T}\end{array}\right.$

Constraint 1 ensures that the sum of the cost of executing $\mathbf{T}$ on $\mathbf{S}$ under the mapping is less than or equal to the payment. Constraints 2 ensures that the tasks can be completed by the deadline $D$. Constraint 3 ensures that each task can be assigned to one CRP. Constraint 4 ensures that each CRP is assigned one task at least. Constraint 5 denotes the integrality requirements for the decision variables.

We propose MIN_COST algorithm to achieve the mapping as showed in Algorithm 1.

\section{Algorithm 1 MIN_COST}

1. Input: $\mathbf{T}=\left\{T_{1}, T_{2}, \ldots, T_{n}\right\}, \mathbf{R}=\left\{C R P_{1}, C R P_{2}, \ldots, C R P_{m}\right\}$, Payment $P$, Deadline $D$

2. Output: Mapping relation between $\mathbf{T}$ and $\mathbf{R}$

3. Initialize payoff matrix $P(i, j)$

4. Initialize Time matrix $T(i, j)$

5. Initialize $k / /$ to record the note of find resource 
6. $\quad$ For $j=1$ to $m$
7. $T_{j}=0$

8. End For

9. For $i=1$ to $n$

10. Initialize $P_{\min }=\operatorname{Max}$

11. For $j=1$ to $m$

12. $\quad$ IF $P(i, j)<=P_{\min } \& \& T_{j}+T(i, j)<=$ Deadline

13. $\quad P_{\min }=P(i, j)$

14. $T P+=P_{\text {min }}$

15. $\quad k=j$

16. Else IF $T_{j}+T(i, j)>D \& \& j==m$

17. Return infeasible mapping

18. End if

19. If $T P>B$

20. Return infeasible mapping

21. End if

22. End for

23. $T_{k}=T_{k}+T(i, j)$

24. End for

25. Return mapping

Our characteristic function in coalitional game is defined as

$v(S)= \begin{cases}P-C(\mathbf{T}, S), & |S|>0 \\ 0, & |S|=0\end{cases}$

Our objective is to find the coalition $S$ such that its members have the highest average global repuation and the coalition provides the maximum individual profit for its members. For the profit sharing among the coalition's members, we will use the equal sharing method, which provides a tractable way to determine the shares.

Due to their welfare-maximizing behavior, the CRPs prefer to form a low profit coalition if their profit divisions are higher than those obtained by participating in a high profit coalition. Also, the CRPs will prefer a coalition with the highest average reputation for its member. So, a CRP $R$ determines its preferred coalition $S$, by solving:

$\left\{\begin{array}{l}\max _{S} \frac{P-C(\mathbf{T}, S)}{|S|} \\ \max _{S} \frac{\sum_{i: R_{i} \in S} r_{i}}{|S|}\end{array}\right.$

Where $r_{i}$ is the reputation value of $R_{i}$.

Obviously, this is a bicriteria optimization problem in which the CRP's goal is maximize the profit sharing that it obtains from the coalition and at the same time maximize the average global trust it has within the coalition. Minimizing the cost $C(\mathbf{T}, S)$ by MIN_COST implicitly maximizes the profit, $P-C(\mathbf{T}, S)$, earned by a coalition. That means, a coalition finds the maximum profit, then the profit is divided among all members. As a result, a CRP prefers a coalition that provides the highest profit among all possible coalitions.

Algorithm 2 is used to decide the coalition formation structure. Algorithm 3 starts by assigning the same reputation score to all CRPs in the coalition $S$. Then, it recomputes the reputation scores of each CRP as the weighted sum of the scores of all CRPs in a CRP's neighborhood. The algorithm repeats these steps until the average relative error between $\mathbf{r}^{l+1}$ and $\mathbf{r}^{l}$ is smaller than the given threshold $\zeta$. That means $\mathbf{r}^{l}$ does not change 
significantly any more and it represents the global reputation of the CRPs in $S$. The algorithm returns the eigenvecting the reputation of CRPs participanting in the coalition.

Algorithm 2 TCFA: Trust-based Coalition Formation Algorithm

1. Input: $\mathbf{R}$

2. Output: Coalition structure

3. Initialize the set of feasible CRPs coalitions, $F=\varnothing$ and the set $S$ with the set of all CRPs, $S=\mathbf{R}$

4. Call MIN_COST algorithm to find the mapping $\pi_{S}: \mathbf{T} \rightarrow S$

5. If step 4 is completed, $F \leftarrow S \cup F$

6. Call algorithm 2 to compute the reputation values of all CRPs in coalition

7. Find a CRP R with the lowest reputation in $\mathbf{x}$

8. $S \leftarrow S \backslash R$

9. Find $k=\arg \max _{S i \in F}\left\{v\left\{S_{i}\right\} /\left|S_{i}\right|\right\}$

10. Return mapping and execute $\mathbf{T}$ on coalition $S_{k}$

Algorithm 3 Reputation computing

1. Input: Trust graph $(S, \Xi)$

2. Output: $\mathbf{r}$

3. Compute $A_{S}=$ adjacency matrix of $(S, \Xi)$

4. Compute $r_{R}^{0} \leftarrow 1 /|S|$ for all $R_{i} \in S$

5. Repeat

6. $\quad \mathbf{r}^{l+1} \leftarrow A_{S}^{T} \mathbf{r}^{l}$

7. $\quad \theta \longleftarrow\left\|\mathbf{r}^{l+1}-\mathbf{r}^{l}\right\|$

8. Until $\theta<\zeta$

9. $\quad$ Return $\mathbf{r}^{l+1}$

\section{Analysis of TCFA}

Theory 1 The coalition structure determined by TCFA is stable.

Proof In our coalition game, the meaning of the stability is that a coalition $S$ is stable if no CRP $R \in S$ can leave $\mathrm{S}$ without making at least one CRP $R^{\prime} \in S$ unhappy. Two cases are considered. First, if $R$ is the CRP that has the lowest reputation among all CRPs in the coalition $S$, then TCFA checks this case by removing $R$. thus, we have two cases where either the coalition is not feasible or the individual profit of CRPs is not as much as the individual profit of CRPs in $S$. As a result, leaving $R$ as part of the coalition makes other CRPs in $S$ unhappy. Second, if $R$ is not the CRP that has the lowest reputation among all CRPs in the coalition $S$, then removing $R$ decreases the total reputation of CRPs in $S$, thus the patrticipating CRPs would be unhappy due to $R$ leaving the coalition. So, the formed coalition determined by TCFA is stable.

Theory 2 There exists the Pareto optimal solution in TCFA.

Proof Based on the definition of Pareto optimality, we need to prove the resulting coalition $S \in F$ from TCFA is not dominated by other coalitions in both its individual payoff $\pi$ and its average reputation $\bar{r}$. Since TCFA removes a CRP with the lowest reputation, the high reputable CRPs are always in the coalition. As a result, the CRPs outside the coalition are not able to form a coalition with higher average reputation than $\bar{r}$. That means, there is no coalition $S^{\prime} \notin F$ where $\bar{r}^{\prime} \geq \bar{r}$. However, there may be other coalition $S^{\prime} \in F$ that have higher average reputation. Those coalitions do not have a higher individual payoff than $\pi$, since TCFA selects $S$ which has the highest individual payoff among all CRPs in $F$. As a result, the coalition formed by TCFA is a Pareto optimal solution. 


\section{Experiments and Results}

In this sector, some simulation experiments are performed for investigating how effective our proposed algorithm is in producing stable CRPs coalition formation. Table 1 gives the parameters and their values setting. CloudSim is selected as the simulation platform of our experiments, which enables modeling and simulation of cloud computing systems and application provisioning environment and can quantify the performance of different scheduling strategies for efficiently use cloud resources [13].

Table 1. Parameter Setting in our Simulation

\begin{tabular}{|l|l|l|}
\hline Parameter & Meaning & Value setting \\
\hline$n$ & Number of cloud tasks & 100 \\
\hline$m$ & Number of CRPs & 30 \\
\hline$M I_{i}$ & Task $i$ 's workload, $\mathrm{i}=1,2, \ldots, n$ & {$[200,400]$, uniform distribution } \\
\hline$M I P S_{j}$ & CRP $j$ 's computing capacity, $j=1,2, \ldots, m$ & {$[100,200]$, uniform distribution } \\
\hline$t$ & Execution time matrix & MI/MIPS, unit:second \\
\hline$C_{i j}$ & Cost matrix & {$[10,50]$, uniform distribution } \\
\hline$D$ & Deadline of tasks & 50, unit:second \\
\hline$P$ & Payoff & 350 \\
\hline
\end{tabular}

Random Coalition Formation Algorithm (RCFA) is selected as the compared algorithm in our experiment analysis. RCFA is similar as our algorithm TCFA, but instead of removing a CRP with the lowest reputation value from a coalition, RCFA removes a CRP without considering its reputation value,i.e., RCFA removes a CRP from a coalition randomly.

Figure 2 shows the completion time of tasks and average global reputation in TCFA and RCFA. We can see that when the number of tasks increases, TCFA will get higher completion time of taks than RCFA. The reason is that TCRA needs to take more time for finding the mapping between tasks and CRPs. But, on the whole, this completion time is reasonable when executing these required tasks. In addition, since TCFA forms coalitions composed of more reputable CRPs, the average global reputation of the members of the coalitions produced by TCFA is higher in all cases then that of TCFA. And TCFA tries to keep the average reputation values the same for all coalitions.

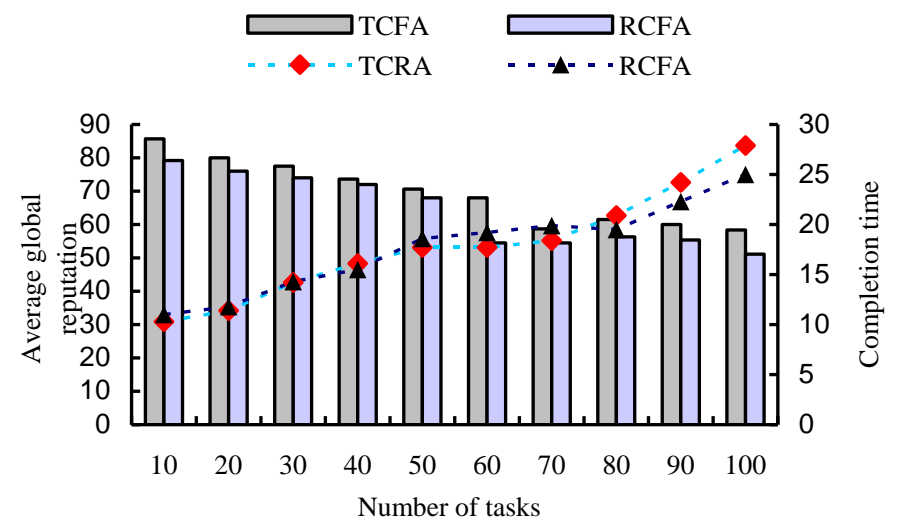

Figure 2. Completion Time of Tasks and Average Global Reputation

Figure 3 shows the changes of individual payoff in the coalition and the number of members in the coalition when the number of executed tasks increases. We can see that the individual payoff for all CRPs in the coalition is same approximately in both algorithms. The reason is that they all select the coalition that yields the highest individual 
payoff for the CRPs. In addition to selecting the coalition yielding the highest individual payoff, our algorithm TCFA also guarantees that the selected coalition is composed of CRPs with highest average global reputation. Meanwhile, this figure also shows as the number of executed tasks increases the number of members in the coalition by TCFA increases, which means that the more tasks the more CRPs pool their resources to form a coalition in order to execute cloud tasks. While the coalitions formed by RCFA do not necessary have smaller size then the coalitions obtained by TCFA.

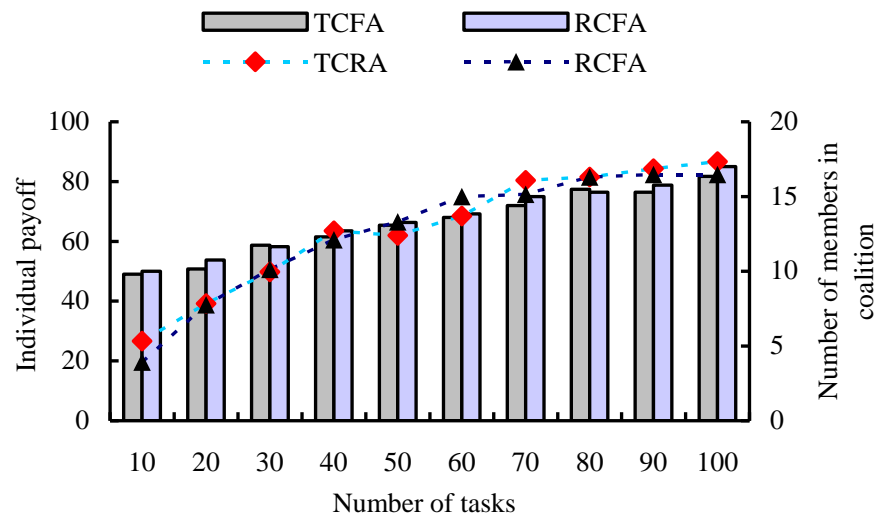

Figure 3. Payoff and Coalitional Size

Figure 4 and Figure 5 show the two optimal objectives in TCFA and RCFA. We can observe that in Figure 4 a coalition with 8 CRPs is the final coalition obtained by TCFA that provides the highest individual payoff and the highest average global reputation. The final coalition does not have the highest average global reputation, but it provides the highest product of individual payoff and average global reputation. In Figure 5 produced by RCFA, the average global reputation also changes but it does not increase since CRPs are removed randomly. Selecting a coalition with highest individual payoff does not provide the highest produce of individual payoff and average global reputation. In addition, TCFA selects the final coalition with the highest individual payoff, but this coalition is the one that also has the highest average reputation. This is because TCFA removes a CRP with the lowest reputation among all CRPs in a coalition in each step of our alogithm.

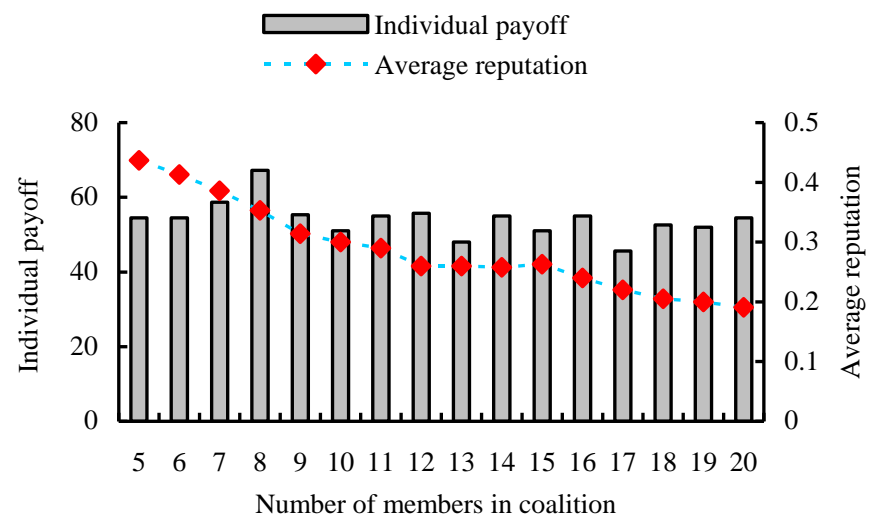

Figure 4. Performance of TCFA 


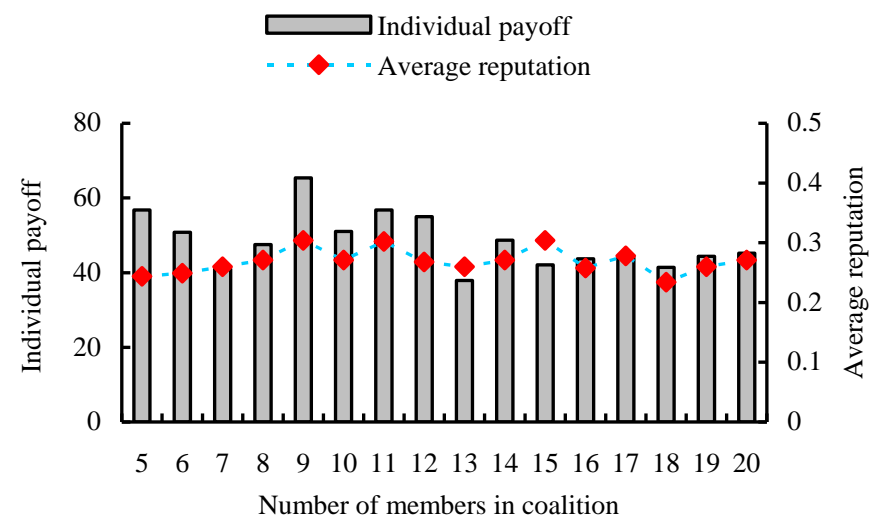

Figure 5. Performance of RCFA

Based on the above results, we conclude our algorithm TCFA can form stable CRPs coalitions, which can guarantee the cloud tasks are completed satistfying all constraints and provide the highest individual payoff for all CRPs in the coalition.

\section{Conclusion}

For obtaining more profit, CRPs can cooperate to form a CRPs coalition in order to execute cloud users' tasks. However, the trust relationships among CRPs have to be considered in this case. In this paper, we model the above problem as a coalitional game and present a novel CRPs coalitions formation algorithm TCFA. TCFA can enable the CRPs with high reputation to join into a coalition reducing the cost of execution and guaranteeing the maximum profit for the participanting CRPs. Furthermore, we prove that the formed coalition determined by our algorithm is stable, which means that the CRPs that are the part of the coalition do not have incentives to break away from it. Meanwhile, we prove that our algorithm can produce a Pareto optimal solution. The results of simulation experiments show that our algorithm not only can produce stable coalitions structure composed of CRPs with higher reputation, but also can obtain higher individual profit among coalitional members.

\section{Acknowledgments}

The work was supported by the project of Science and technology research of henan province (152102210202), Institutions of higher learning key research projects in henan province department of education (15A520094), Science and Technology Innovation Fund of Xinxiang University (15ZA03).

\section{References}

[1] William Voorsluys, James Broberg, Rajkumar Buyya, "Introduction to Cloud Computing, Cloud Computing: Principles and Paradigms", Wiley Press,USA, (2011),pp.1-44.

[2] CHUN L, LA L, "Optimal resource provisioning for cloud computing environement", Journal of Supercomputing, vol. 62, no.2, (2012),pp.989-1022.

[3] Senthil P., Boopal N., Vanathi R., "Improving the security of cloud computing using trusted computing technology", Journal of Modern Engineering Research, vol.2,no.2, (2012),pp.320-325.

[4] Kailash P., Ravindra G., Gajendra S., et.al., "Intergrating the trusted computing platform into the security of cloud computing system", Journal of Advanced Research in Computer Science and Software Engineering, vol.2,no.2, (2012),pp.1-5.

[5] Chunhua H, Jibo L, Jianxun L, "Service selection based on trust evolution and union for cloud computing", Journal of Communication, vol.32,no.7,(2011),pp.71-79.

[6] Wenjuan L, Lingdi P, Qinlong Q,et.al., "Research on trust management strategies in cloud computing environment", Journal of Computational Information Systems, vol.8,no.4,(2012),pp.1757-1763. 
[7] Liu Y.,Wang X, Wang Z, et.al., "Energy-aware and trust-driven virtual machine shceduling", Application Research of Computers,vol.29,no.7,(2012),pp.2479-2483.

[8] Zhang W,Liu X, Yun X,et.al., "Trust-driven job scheduling heuristic for computing grid", Journal of Communications, vol.27,no.2,(2006),pp.73-79.

[9] L.Lin,J.Huai, "Qgrid : an adaptive trust aware resource management framework", IEEE Systems Journal, vol.3,no.1,(2009),pp.78-90.

[10] R. Subrata, A.Y. Zomaya, B. Landfeldt. "A cooperative game framework for QoS guided job allocation schemes in grids". IEEE Transactions on Parallel and Distributed Systems, vol.57, no.10, (2008),pp.1413-1422.

[11] R. Kaewpuang, D. Niyato, P. Wang, and E. Hossain, "A framework for cooperative resource management in mobile cloud computing", IEEE Journal on Selected Areas in Communications-Special Issue on Networking Challenges in Cloud Computing Systems and Applications, vol.31, no. 12, (2013), pp.2685-2700.

[12] D. Niyato, P. Wang, E. Hossain, W. Saad, and Z. Han, "Game theoretic modeling of cooperation among service providers in mobile cloud computing environments", in Proceedings of IEEE Wireless Communications and Networking Coference, (2012), pp.3128-3133.

[13] D. Niyato, Z. Kun, and A. V. Vasilakos, "Resource and revenue sharing with coalition formation of cloud providers: Game theoretic approach", in Proceedings of IEEE International Symposium on Cluster Computing and the Grid, (2011), pp.23-26.

[14] Rodrigo C, Rajiv R, Anton B, et al. "CloudSim: a toolkit for modeling and simulation of cloud computing environments and evaluation of resource provisioning algorithms", Software: practice and experience, vol.41, no.1, (2011), pp.23-50.

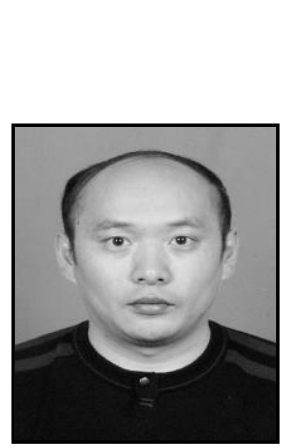

\section{Authors}

Lijun Xu, he is currently a Ph.D. student in the School of Computer Science and Technology at Wuhan University of Technology and an associate professor at the Xinxiang University. He received his B.S. from Henan Normal University, Xinxiang, Henan, China, in 2001, and M.S. from the Wuhan University of Technology, Wuhan, Hubei, China, in 2007. He has worked at the Xinxiang University since 2001. His research interests include resource management in cloud computing environments and Computer network. He has published about 10 papers. 
International Journal of Grid and Distributed Computing Vol. 9, No. 11 (2016) 